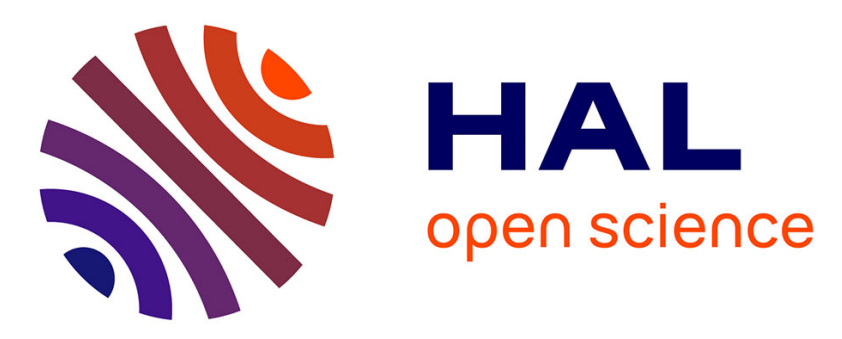

\title{
Affective, Social, and Cognitive Outcomes During a 1-Year Wintering in Concordia
}

\author{
Michel Nicolas, Peter Suedfeld, Karine Weiss, Marvin Gaudino
}

\section{To cite this version:}

Michel Nicolas, Peter Suedfeld, Karine Weiss, Marvin Gaudino. Affective, Social, and Cognitive Outcomes During a 1-Year Wintering in Concordia. Environment and Behavior, 2015, 48 (8), pp.1073 - 1091. 10.1177/0013916515583551 . hal-01634242

\section{HAL Id: hal-01634242 \\ https://hal.science/hal-01634242}

Submitted on 13 Nov 2017

HAL is a multi-disciplinary open access archive for the deposit and dissemination of scientific research documents, whether they are published or not. The documents may come from teaching and research institutions in France or abroad, or from public or private research centers.
L'archive ouverte pluridisciplinaire HAL, est destinée au dépôt et à la diffusion de documents scientifiques de niveau recherche, publiés ou non, émanant des établissements d'enseignement et de recherche français ou étrangers, des laboratoires publics ou privés. 


\title{
Affective, Social, and Cognitive Outcomes During a I-Year Wintering in Concordia
}

\section{Michel Nicolas', Peter Suedfeld², Karine Weiss ${ }^{3}$, and Marvin Gaudino'}

\begin{abstract}
This study investigated time patterns and the relationships between perceived stress, recovery, control, attention lapses, and defense mechanisms (DM) during a 12-month wintering in Concordia polar station with an international crew of 14 volunteers. This ICE (Isolated, Confined, Extreme) environment induced some stress, mainly in the social dimension and showed relationships (a) between DM and both stress and recovery and (b) between recovery and perceived control, highlighting the roles of DM and control in psychological adaptation. These results offer additional insights into the affective, social, and cognitive processes involved in adaptation. The findings suggest that preventive psychological countermeasures should be developed for crew members to counteract detrimental psychological outcomes and to improve adaptation to long-duration ICE situations such as planned human interplanetary space missions.
\end{abstract}

\section{Keywords}

affect, cognition, social, adaptation, ICE environment

\footnotetext{
'University of Burgundy, Dijon, France

2University of British Columbia, Vancouver, Canada

3University of Nîmes, France
}

\section{Corresponding Author:}

Michel Nicolas, Laboratory of Socio Psychology and Management of Sport (SPMS, EA 4180), Faculty of Sport Sciences - UFR STAPS, University of Burgundy, University Campus, BP 27877, Dijon Cedex 21078, France.

Email: michel.nicolas@u-bourgogne.fr 


\section{Introduction}

Despite centuries of widespread exploration, human knowledge about many aspects of life in different parts of the Earth is incomplete and is still the subject of active investigation. Beyond our planet and its immediate surroundings, the next great step for humanity will certainly be the planned long-term space mission to Mars, which raises many questions about human adaptation. Psychological Adaptation Processes (PAPs) are among the key issues for the success of such a mission (Palinkas \& Suedfeld, 2008), and psychosocial factors are now recognized as critical components of mission success (Bishop, 2004; Manzey \& Lorenz, 1998). Space analogues such as Antarctic stations are recognized as natural laboratories for the study of the effects of isolation and confinement on human behavior (Suedfeld \& Weiss, 2000).

The international Antarctic Concordia Station offers one of the most severe environments on Earth in which to study the effects of long-term isolation and confinement on healthy volunteers and their adaptation processes. During a winter in Concordia Station, participants face unfamiliar psychological challenges arising from both psychosocial and physical origins. Crew members have little control over their own schedules, which tend to fluctuate between periods of idle boredom and periods of intense work. They must cope with a drastically unusual social dimension, contacts with family and friends at home are seriously disrupted, with limited telecommunications. Within the station, they face low levels of privacy and almost constant forced social interaction with the same small and unchanging group; in some stations, communication with crewmates is hampered by the lack of a common culture and native language.

The physical environment is also novel and challenging, especially during the Antarctic winter, when even emergency medical evacuation may be impossible. The crew is isolated and confined in a site far from their accustomed surroundings. The natural environment is harsh and often dangerous. It is marked by unusual light-dark cycles, low levels of atmospheric oxygen, low humidity, extreme cold, and sudden storms (Salam, 2012). The indoor environment is monotonous and offers limited mobility and stimulation. Architecture and interior design emphasize efficiency, ease of transport, and cost, not comfort or esthetic quality. Previous research has shown that ICE (Isolated, Confined, Extreme) stations in Antarctica can induce adverse effects, including dysfunctional responses to stress, fatigue, lack of energy, conflicts and tensions, perceived loss of control, and decrements in attention and cognitive functioning (Leon, Sandal, \& Larsen, 2011).

However, according to the seminal models of stress, below a certain threshold stress is considered to be necessary for adaptation both biologically 
(Selye, 1950) and psychologically (Lazarus \& Folkman, 1984). Stress responses in ICE environments have been reported to induce excitement or distress, depending on the stress level (e.g., Marsh \& Rygalov, 2008). An appropriate level of stress can improve adaptive responses to constraining or extreme situations (Palinkas, 1992). However, excessive levels or ineffective regulation of stress in ICE could generate dysfunctional adaptation, with detrimental outcomes both in crews' well-being and performance (Geuna \& Brunelli, 1995; Nicolas \& Gushin, in press; Nicolas \& Weiss, 2009). For organizations involved in Antarctic operations, such knowledge of potential stressors (physical, emotional, social, and behavioral), recovery regulation, and defense mechanisms (DM) would be useful in two ways: to improve the selection of personnel and to identify countermeasures that could help crews cope with these constraining conditions.

Stress responses have been well documented in space missions and simulations, although there is still a need for investigations into the development and relationships among the multidimensional aspect of ICE stress (Suedfeld, 2005). Coping and recovery resources are an even more neglected phenomenon in ICE research (Nicolas \& Weiss, 2009), in spite of the demand/ resource balance necessary to adjust to the stressors typical of those environments (Lazarus, 2006). The recovery process involves the restoration of physiological and psychological resources (Kellmann \& Kallus, 2001). Thus, the restoration of resources can counterbalance negative effects of stress and thus help crew members to adjust to the situation.

Models of recovery from stress, such as that of Kellmann and Kallus (2001), can indicate the impact of various sources of stress and to compare perceived stress levels to the person's own capabilities to recover. Studies of sport psychology (Kellmann \& Günter, 2000) and space analogue environments (Nicolas \& Weiss, 2009) have shown the effects of a deficit in recovery. Such deficits can interfere with performance and also induce decrements in emotional, cognitive, or behavioral outcomes. These, in turn, can compromise the participant's adaptation to environmental demands and constraints.

Long-term sojourns in a polar station can produce cognitive decrements such as lapses of attention (Kanas et al., 2009; Palinkas \& Suedfeld, 2008). Beyond mere absentmindedness, prolonged lapses of attention are a familiar occurrence in Antarctic stations. Colloquially known as "Long Eye," the $10-\mathrm{m}$ stare in the 5-m room, and depending on their duration, they may interfere with safe and efficient task performance as well as reducing the motivation to persist in and enjoy necessary tasks (Cheyne, Carriere, \& Smilek, 2006). In ICE environments, some disruptions of attention can cause accidents, injury, and loss of life. During the 1989 winter season at McMurdo Station, $51.5 \%$ of residents reported difficulties with concentration or 
memory (Palinkas, 2003). Such symptoms are most frequently attributed to fatigue, low levels of environmental stimulation, and neurobehavioral effects of exposure to cold (Palinkas \& Suedfeld, 2008).

DM are theoretically recognized as major constructs in psychological adaptation such as coping strategies (CS; Cramer, 1998; Parker \& Endler, 1996). Indexed in the Diagnostic and Statistical Manual of Mental Disorders since its fourth edition (DSM-IV; American Psychiatric Association, 1994), DM are defined as automatic psychological processes that protect the individual against anxiety and from the awareness of internal or external dangers or stressors. DM are considered to be related to personality as a framework of disposition-based responses. In contrast, CS are more determined by situation-specific variables (Cramer, 1991; Nicolas \& Jebrane, 2008b; Parker \& Endler, 1996). Several empirical studies have shown that DM are involved in, and contribute to, adjustment processes in the general population (Bouchard \& Thériault, 2003; Erickson, Feldman, \& Steiner, 1997; Fulde, Junge, \& Ahrens, 1995) as well as in athletes engaged in competition (Nicolas \& Jebrane, 2008a). Despite the important theoretical and practical issues at stake, there have been few, if any investigations of the functioning of DM, in extreme ICE situations.

PAPs have received a fair amount of scientific attention. Nevertheless, there is a need for greater knowledge of the multidimensional patterns of change in both stress and recovery over time. Even less research has addressed the relationships of these patterns to personality-based DM and cognitiverelated attention lapses. The aim of the current study was to investigate the affective, social, cognitive, and personality aspects of the adaptation process. Relevant aspects of that process include the time-course of stress, recovery and control states, DMs, and lapses of attention.

The study focused on these aspects, and the relationships among them, during a 1-year Antarctic wintering by healthy volunteers, defined as "winterers" to differentiate them from the more numerous summer staff and visitors. The site was Concordia Station on the Antarctic Continent; for details, see "Concordia Station Overview and Facilities" section. Based on the findings cited previously, we expected that the participants would report increased levels of stress indicators and decreased levels of recovery factors during the ICE period compared with baseline levels. In addition, we expected to find relations among stress, recovery, control, DM, and lapses of attention.

\section{Method}

\section{Participants}

The crew consisted of 14 winterers ( 1 woman and 13 men, 8 French and 6 Italians), ranging in age from 21 to 58 years of age $(M=38.14, S D=11.90)$. 
Winter-over crews for Concordia are selected through specific medical and psychological screening. After the selection process, the participants were given oral and written explanations of the study objectives, procedures, and the potential risks of participation. The participants gave their written informed consent to participate in this study, which was approved by the ethics committees of the local university and the European Space Agency. The crew included technicians (for station infrastructure), scientists (to conduct research programs), a cook, and 2 medical doctors. One of the latter was primarily in charge of health issues, while the principal role of the other was the coordination of the research programs, including the research reported in this article.

\section{Instruments}

Recovery-Stress Questionnaire (RESTQ-48). The RESTQ-48 (Kellmann \& Kallus, 2001) was used to assess the balance between stress and recovery states in a multilevel approach. From the perspective of a biopsychological stress model, the RESTQ includes general dimensions concerning stress and recovery as well as specific dimensions. These address more details of the stress and recovery processes from physical (somatic), emotional, behavioral, and social perspectives.

For the stress dimension, items include "I felt physically bad" and "I felt under pressure." For the recovery dimension, items include "I felt physically fit" and "I felt at ease." The Total Stress dimension score corresponds to the mean scores on the seven stress subscales (General Stress, Emotional Stress, Social Stress, Conflicts/Pressure, Fatigue, Lack of Energy, and Somatic Complaints). The Total Recovery dimension score is derived from the mean of scores on the five recovery subscales (Success, Social Relaxation, Somatic Relaxation, General Well-Being, and Sleep Quality). The internal consistency for Total Stress and Total Recovery was within the acceptable range, respectively, .88 and .90 .

Perceived Stress and Control and Mastery Scales. In accordance with the central tenets of stress theory (S. Cohen, 1978; Lazarus, 1966, 1977), the questions in the Perceived Stress Scale (PSS; S. Cohen \& Williamson, 1988) for perceived stress, and in the Mastery scale (Pearlin \& Schooler, 1978) for perceived control, are relatively independent of content that would be specific to any particular situation and population. For a short and quick administration, four items for perceived stress and four items for perceived control were reworded. The revision made the items relevant to the specific ICE situation, to measure the degree to which this environment is appraised as stressful and 
controllable. For this sample, the internal reliability estimates for perceived stress and control were, respectively, .79 and .68 .

Defense Style Questionnaire (DSQ-40). The 40-item version of the DSQ-40 (Andrews, Singh, \& Bond, 1993) assesses an individual's conscious derivatives of DM in terms of DSM concepts, classification, and nomenclature. For this study, we used the two higher order Mature and Immature defense styles that had been shown to be involved in extreme or constraining situations (Nicolas \& Jebrane, 2008a, 2008b; Nicolas, Sandal, Weiss, \& Yusupova, 2013). Mature defenses are the tendency to use problem-solving styles, whereas Immature defenses are the tendency to express affect through reactions of behavioral withdrawal or acting-out. DSQ-40 possesses reasonable psychometric properties, including internal consistency and temporal stability when used in clinical and research settings (Muris \& Merckelbach, 1996). In the present study, alpha coefficients were .72 for the Mature factor and .71 for the Immature factor.

Attention lapses. Participants completed 5 items extracted from the 15 -items Mindful Attention Awareness Scale (MAAS; Brown \& Ryan, 2003), selected as a measure of attention lapses. These items measure the ability to sustain conscious awareness of attention (e.g., "I find it difficult to stay focused on what's happening in the present"; "I find myself doing things without paying attention"). They were chosen as general statements adaptable to ICE situations. MAAS scores are based on the mean of all items and can range from 1 to 6 . Higher scores indicate greater mindfulness. The internal consistency of these 5 items was within an acceptable range, Cronbach's $\alpha=.72$.

\section{Procedure}

Each questionnaire, as adapted for the ICE environment, was translated from the original English version to French and Italian, using a back-translation procedure. A bilingual scientist translated the scales, and another bilingual scientist then translated them back into English. The translated scales were first administered to three French and three Italian participants to identify any unclear items. Based on the ratings and comments provided by the participants, a small number of items were closely examined and reworded to improve clarity.

The study covered a 12-month period. Potential participants received an explanation of the purpose and the procedure of the study and gave their informed consent during the pre-departure seminar at the French polar institute (IPEV, Institut Paul Emile Victor, Plouzané, France). Each questionnaire, as adapted to the ICE situation and then translated, was explained to the crew. 
During the winter, questionnaires were sent to each volunteer via the Internet at scheduled times. To ensure the confidentiality of the data, participants returned the completed questionnaires directly to the researchers. All of the questionnaires ask the respondent to indicate the extent to which each statement corresponds to his or her perception, using a Likert-type scale anchored by $1=$ never and $6=$ always. The first (baseline) administration of the psychological measures (ICE 1) occurred within the first day of the deployment. Other administrations occurred throughout the wintering deployment at +3 months (ICE 2 ), +5 months (ICE 3 ), +6 months (ICE 4$),+8$ months (ICE 5), +10 months (ICE 6), and +12 months (ICE 7).

\section{Concordia Station Overview and Facilities}

Opened in November 2004, Concordia polar station is one of the three permanent stations on the high Antarctic plateau. The others are the Russian Vostok and the American Amundsen-Scott Stations. Concordia Station was built and is jointly operated by the French Polar Institute (IPEV) and the Italian Antarctic Research Programme (PNRA). The European Space Agency (ESA) entered into cooperation with IPEV and PNRA mainly to coordinate research in glaciology, atmospheric sciences, astronomy and astrophysics, earth sciences, technology, and health. The ESA's Directorate of Human Life Sciences is in charge of Concordia's programs in medicine, physiology, and psychology.

Concordia Station is a prototypical ICE environment. It is located at altitude 3,232 $\mathrm{m}$ at Dome C $\left(75^{\circ} 06^{\prime} \mathrm{S}, 123^{\circ} 23^{\prime} \mathrm{E}\right), 1,000 \mathrm{~km}$ inland from the coast of the Antarctic Ocean. The high Antarctic mainland is one of the coldest (mean temperature of $-51^{\circ} \mathrm{C}$, the lowest recorded $-85^{\circ} \mathrm{C}$ ), driest, most inhospitable and inaccessible regions on the planet. Access to the station is only possible during the Antarctic summer, from mid-November to mid-February.

Concordia shares many of the features described in the introductory analysis of ICE environments. The situation involves several stressors and challenges to the overwintering crews, including a fascinating but dangerous natural environment, with extremely low outside temperatures, strong and almost constant wind, and a flat white landscape. Its atmosphere is characterized by chronic hypobaric hypoxia: an altitude equivalent to almost $4,000 \mathrm{~m}$ on the equator, with air pressure of about $645 \mathrm{hPa}$. The crew needs to be totally self-dependent. This is especially true from February to November, when no access to or from Concordia Station is possible, even in emergencies. The living and working quarters are confined to small two-story buildings. The crew is multicultural with different first languages, traditions, and 
customs. They have limited access and mobility outside the station buildings, especially during winter, and consequently experience long periods of understimulation and boredom. The strangeness of the environment is increased by the very long polar nights and days, characterized by continuous light during the Antarctic summer and prevalent darkness during winter.

\section{Statistical Analyses}

Given that the scores on all factors were normally distributed, data were analyzed using parametric statistics. Changes in stress and recovery states were first assessed using repeated analyses of variance in comparison with baseline measure. Afterwards, post hoc analyses with paired $t$ tests were applied to determine which specific values were significantly different. In addition, relationships among the variables were tested using the Pearson correlation coefficient $(r)$. The level of significance for these analyses was corrected using Bonferroni-type adjustment to maintain the probability of Type I error at the .05 alpha level $(p<.008)$. Effect sizes (ESs) were calculated as the difference between the means divided by the standard deviation of the difference. As a general guideline, an ES of $0.20-0.50$ is considered to be a small effect, one of $0.50-0.80$ a medium effect, and a value $>0.80$ a large ES (J. Cohen, 1988). Internal consistency of all scales was assessed using Cronbach's alpha coefficient. Means and standard deviations for all scales at each administration are reported in Table 1.

\section{Results}

\section{Changes in Stress-Recovery States, DM, Perceived Stress and Control, and Attention Lapses Over Time}

Total stress showed significant variations during the 12-month wintering (see Table 1). Mean scores on Total Stress, $F(6,48)=5.02, p<.001, d=1.58$ (Figure 1) showed a significant increase throughout the wintering for all participants, due entirely to changes in the social dimension of stress, $F(6,48)=$ $4.20, p<.002, d=1.45$ (see Figure 1). Changes in the other stress scale dimensions were not statistically significant. None of the changes in the recovery dimensions reached statistical significance: Total Recovery, $F(6,48)=1.45$, as well as all its components.

Post hoc analyses showed significant variations only with the baseline measure ICE 1. Compared with the baseline measure at the beginning of the wintering (ICE 1), Total Stress showed a progressive and significant increase during the ICE period. More specifically, the total mean of subjective stress 
Table I. Means of Total Stress, Social Stress, Total Recovery, Perceived Stress and Control, DM Mature and Immature, and Attention Lapses Scales During I-Year Wintering in Which Winterovers Confront ICE.

\begin{tabular}{|c|c|c|c|c|c|c|c|}
\hline & ICE I & ICE 2 & ICE 3 & ICE 4 & ICE 5 & ICE 6 & ICE 7 \\
\hline \multicolumn{8}{|c|}{ Total Stress } \\
\hline$M$ & I.7I & $2.03 *$ & $2.08 *$ & $2.14^{*}$ & $2.18^{*}$ & $2.28^{*}$ & $2.21^{*}$ \\
\hline$S D$ & 0.35 & 0.36 & 0.45 & 0.38 & 0.66 & 0.75 & 0.48 \\
\hline \multicolumn{8}{|c|}{ Social Stress } \\
\hline$M$ & 1.59 & $1.95 *$ & $2.04 *$ & $1.99 *$ & $2.18 *$ & $2.53^{*}$ & $2.48^{*}$ \\
\hline$S D$ & 0.36 & 0.54 & 0.76 & 0.55 & 0.88 & 0.97 & 0.78 \\
\hline \multicolumn{8}{|c|}{ Total Recovery } \\
\hline$M$ & 4.41 & 4.51 & 4.28 & 4.17 & 4.30 & 4.11 & 4.19 \\
\hline$S D$ & 0.64 & 0.57 & 0.57 & 0.46 & 0.59 & 0.60 & 0.68 \\
\hline \multicolumn{8}{|c|}{ Perceived Stress } \\
\hline$M$ & 1.48 & 1.68 & $1.93^{*}$ & $2.10 *$ & 1.79 & $2.21 *$ & $2.18^{*}$ \\
\hline$S D$ & 0.58 & 0.68 & 0.80 & I & I & 1.06 & 0.90 \\
\hline \multicolumn{8}{|c|}{ Perceived Control } \\
\hline$M$ & 3.32 & 3.45 & 3.32 & 3.36 & 3.40 & 3.39 & 3.49 \\
\hline$S D$ & 0.40 & 0.50 & 0.34 & 0.48 & 0.48 & 0.66 & 0.58 \\
\hline \multicolumn{8}{|c|}{ DM Immature } \\
\hline$M$ & 1.96 & 1.91 & 1.90 & 1.92 & 1.87 & 1.97 & 1.91 \\
\hline$S D$ & 0.41 & 0.38 & 0.34 & 0.41 & 0.36 & 0.34 & 0.40 \\
\hline \multicolumn{8}{|c|}{ DM Mature } \\
\hline$M$ & 3.59 & 3.74 & 3.65 & 3.89 & 3.64 & 3.63 & 3.64 \\
\hline$S D$ & 0.69 & 0.78 & 0.82 & 0.79 & 0.95 & 0.70 & 1.03 \\
\hline \multicolumn{8}{|c|}{ Attention Lapses } \\
\hline$M$ & 1.58 & 1.68 & 1.68 & 1.83 & 1.83 & $1.91 *$ & $2.00 *$ \\
\hline$S D$ & 0.50 & 0.49 & 0.48 & 0.48 & 0.72 & 0.73 & 0.57 \\
\hline
\end{tabular}

Note. For all the scales, $p$ level $<.05$ with Bonferroni correction. DM = defense mechanisms; ICE $=$ isolated and confined environment.

*Significantly different from ICE I.

increased significantly between ICE 1 and ICE $2, t(9)=-3.77, p<.01, d=$ -1.19 ; ICE 3, $t(9)=-5.03, p<.001, d=-1.59$; ICE 4, $t(9)=-7.12, p<.0001$, $d=-1.06$; ICE $5, t(10)=-3.92, p<.01, d=-1.13$; ICE $6, t(8)=-3.54, p<$ $.01, d=-0.74$; and ICE 7, $t(8)=-3.53, p<.01, d=-0.37$. This increase was replicated in the social stress dimension. A progressive and continuing increase with significant differences was found between ICE 1 and ICE 2, $t(9)=-3.42, p<.01, d=-1.08$; ICE 1 and ICE 3, $t(9)=-3.14, p<.05$, $d=-1.11$; ICE 1 and ICE $4, t(9)=-2.90, p<.01, d=-1.09$; ICE $5, t(10)=$ $-3.20, p<.01, d=-1.11$; and the two last and highest measures at ICE 6, 


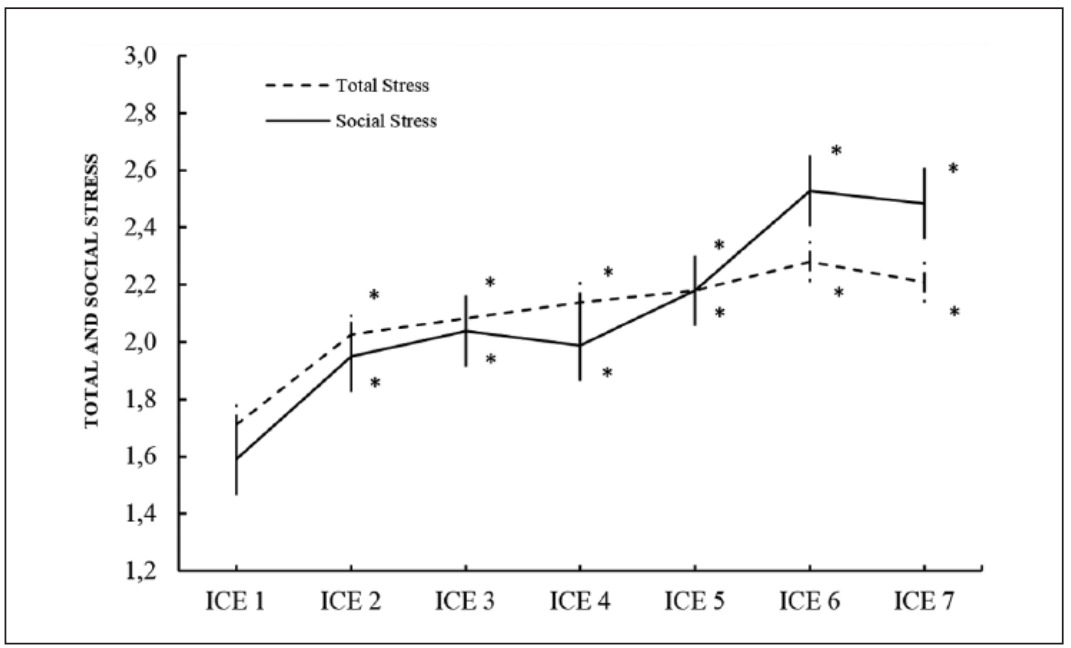

Figure I. Time course of total and social stress throughout the experiment and during the whole wintering in ICE.

Note. $p$ level $<.05$ with Bonferroni correction. ICE $=$ isolated and confined environment. *Significantly different from ICE I for both total and social stress.

$t(8)=-3.94, p<.01, d=-1.47$; and ICE 7, $t(8)=-4.41, p<.01, d=-0.63$. Significant increases were also found for Perceived Stress between ICE 1 and ICE 3, $t(8)=-3.46, p<.01, d=-0.76$; ICE $4, t(10)=-2.73, p<.05, d=$ -0.76 ; ICE $6, t(9)=-3.59, p<.01, d=-1.13$; and ICE 7, $t(8)=-3.46, p<$ $.05, d=-1.15$. Attention Lapses and Concentration also increased between baseline and ICE $6, t(9)=-2.30, p<.05, d=-.73$, and ICE $7, t(8)=-3.13$, $p<.05, d=-1.04$.

\section{Relationships Between Stress-Recovery States, DM, Perceived Stress and Control, and Attention Lapses}

Correlational analyses between stress-recovery states, DM, symptoms of depression, and lapses of attention-concentration indicated several significant relationships (see Table 2). Throughout the wintering, the mean of Total Stress was significantly and positively associated with the mean scores on the PSS $(r=.63)$, Attention Lapses $(r=.83)$, and Immature DM $(r=.71)$. Immature DM were also related to Conflict-Pressure Stress $(r=.83)$ and to Social Stress $(r=.62)$. Total Recovery was positively correlated with Mature DM $(r=.68)$ and Perceived Control $(r=.77)$. Perceived Control was also related to Well-Being $(r=.87)$ and Success $(r=.84)$. 


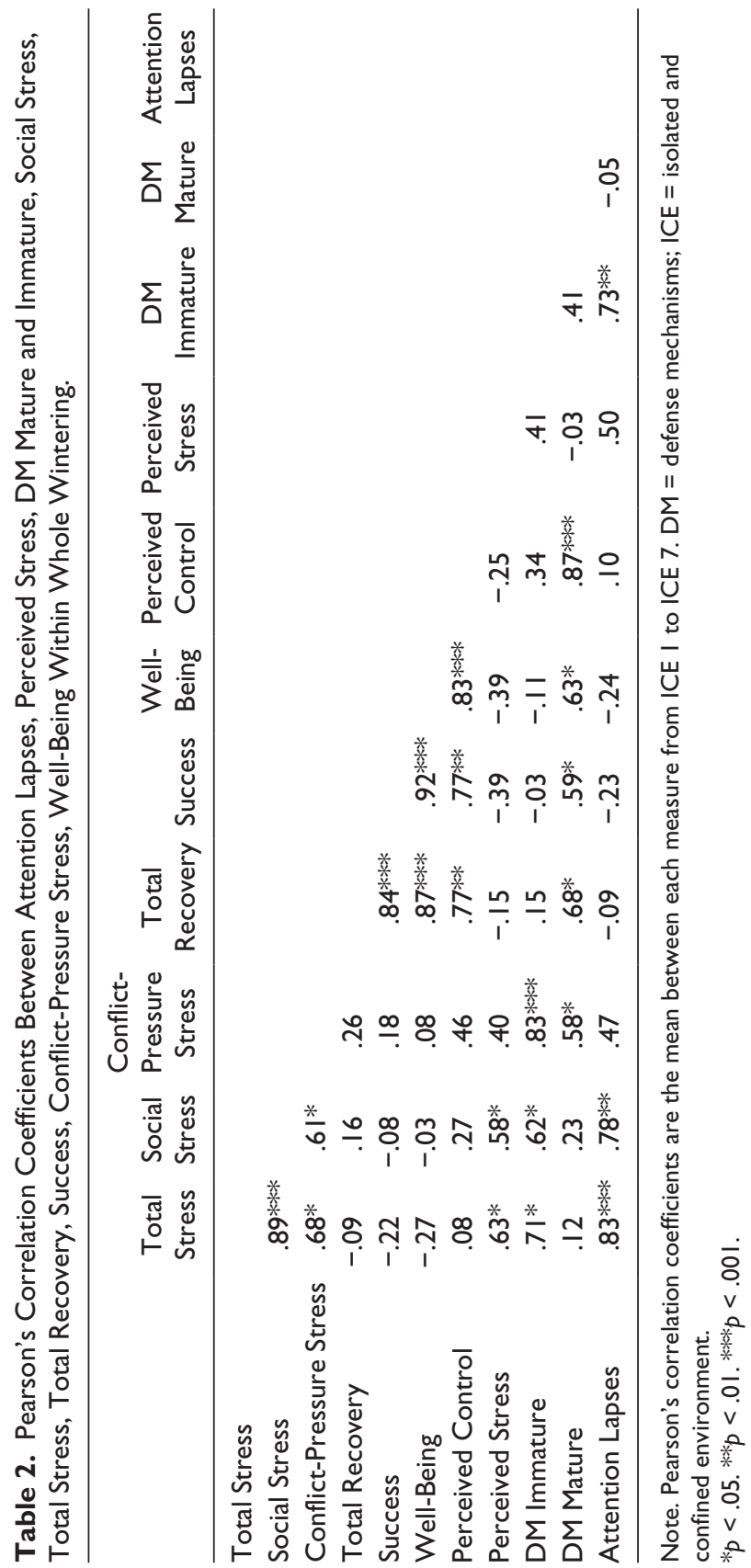

Downloaded from eab.sagepub.com by guest on April 23, 2015 


\section{Discussion}

This study investigated the roles of perceived stress, recovery, control, DM, and attention lapses in the process of psychological adaptation to wintering in Concordia Antarctic Station. Among the effects of stressors, we found a preto post-deployment increase only in the social dimension of stress. It appears that even under such severe and prolonged conditions, ICE environments may have specific rather than general effects on psychological states. However, short-term changes between repeated administrations were found on a few other dimensions of stress.

Relations among stress, recovery, and control states, DM, and attention lapses were also found. Total Stress was associated with Perceived Stress, Immature Defense, and Attention Lapses. Recovery was linked to Perceived Control and Mature Defense. Mature Defense was also associated with the Well-Being and Success recovery scales and Perceived Control. Highlighting the role of defense and control in PAPs, these findings show the impact of an ICE situation on affective and cognitive states and the relations (a) between defense and both stress and recovery and (b) between recovery and perceived control.

\section{Changes in Stress-Recovery States, DM, Perceived Stress and Control, and Attention Lapses Over Time}

Supporting our hypothesis, longitudinal analyses indicated that the total mean of stress increased significantly and progressively throughout the wintering. This increase in subjective stress suggests that the winterers' experience was distressing throughout the wintering and especially at the end of the ICE. Our data further confirm previous studies that have reported that ICE situations can induce psychological stress (Chouker, 2012; Grigor'ev \& Fedorov, 1996; Ishizaki et al., 2002; Nicolas, 2009).

The multidimensional assessment of stress and recovery states at the same time is one of the interests of this research. The Social Stress scale was the only dimension significantly impacted by the strict ICE condition imposed by a wintering in Concordia. Social stress revealed a significant continuous increase across the 12-month ICE. Several studies of small groups in other isolated environments suggest that prolonged isolation and confinement leads to interpersonal issues and increased social tension (for reviews, see Leon et al., 2011; Palinkas \& Suedfeld, 2008; Zimmer, Cabral, Borges, Côco, $\&$ Hameister, 2013). These findings are also in agreement with other recent studies in space analogues, the WISE 2005 study, a 60-day head-down tilt long-term bed rest (HDTBD; Nicolas, 2009), and the Mars 105 experiment 
(Nicolas \& Gushin, in press). Both showed that the social dimension is a key factor in adaptation, especially during prolonged ICE missions that pose difficulties for the participants. During the later stages of such missions, psychosocial problems become increasingly likely.

Complex social interactions may become more problematic in such cases because of several factors. Among these are social comparisons and competition, ostracism and exclusions, cliques, differences in gender, age, occupation, and culture ineffective leadership, restricted and prolonged social contacts, and separation from the usual social network of family and friends (e.g., Kanas et al., 2009; Leon et al., 2011; Stuster, Bachelard, \& Suedfeld, 2000; Suedfeld, 2010). As a psychologist who conducted debriefing interviews at the end of winterings in Antarctic and sub-Antarctic polar stations, the first author found that generally winterers are aware that they had had to adapt to many of the less-than-pleasant and transitory aspects of Antarctic life. However, they have also often reported that interpersonal conflicts and negative experiences within a small crew may be relatively limited, and that they often experienced social cohesion and specific relationships because they shared a common experience (Palinkas, 2003; Palinkas \& Suedfeld, 2008). Studies of Antarctic winterover crews have found that group cohesion and other interpersonal issues are not systematic and vary from one year to another (Palinkas, 1992). However, psychosocial issues are likely to become increasingly important as human beings travel further from Earth on longterm space missions.

\section{Relationships Among Stress, Recovery, Control, DM, and Attention Lapses}

As hypothesized, the findings showed significant relationships among perceived stress, recovery, control, and DM. On one hand, significant positive correlations were found between Total Stress and Perceived Stress, Immature Defense, and Attention Lapses. On the other hand, Total Recovery was positively associated with Perceived Control and Mature Defense, which was also related to Well-Being and Success. Total Stress was related to the PSS, showing convergent validity for this study. Furthermore, Total Stress was also associated with Attention Lapses, indicating that one effect of a prolonged ICE situation may be reduced attention capacity and vigilance. These, in turn, may negatively affect adaptation and task performance. Cognitive alterations in attention could be due to multiple influences such as stress and fatigue, sleep disturbances, or maladaptive reactions to the environment, as well as individual characteristics, low environmental stimulation, and exposure to cold 
(Palinkas \& Suedfeld, 2008; Zimmer et al., 2013). It is important to note that not all studies have found such a cognitive decline (John Paul, Mandal, Ramachandran, \& Panwar, 2010).

Another interesting relationship is that between Total Recovery and Perceived Control. Perceived Control was originally defined as one's need to demonstrate competence and mastery over the environment (White, 1959). Perceived control may be a function of the extent to which the individual perceives that he or she has personal resources, such as requisite skills and abilities, that are adequate for dealing with problems (for a review, see, Armitage \& Conner, 2001). This could mean that individuals who feel that they have the resources to face the demands of ICE life do in fact recover better from this constraining situation.

The process of psychological adaptation can be viewed as a process of negotiation between the individual and the social and physical environment (Palinkas, 1992). In that sense, stressful experiences could lead to the development of new adaptive resources in winterovers, based on some combination of compromise and control. Thus, stressful conditions in the environment may serve as stimuli for adaptive responses (Lazarus, 1999), whereas a lack of control over the social and physical environment could compromise the PAP.

A noteworthy finding of the present study is the role of DM in both stress and recovery processes. Immature Defenses were linked to Stress, and Mature Defenses were associated with Recovery. Previous studies in other constraining situations have shown the link of DM to personality and performance in a sport competition (Nicolas \& Jebrane, 2008a, 2008b) and in a space analogue (Nicolas et al., 2013), confirming the role of DM in psychological adaptation to challenging situations. In line with theoretical frameworks (Cramer, 1998; Parker \& Endler, 1996), these findings point out that DM should be taken into account in selecting personnel with optimal characteristics for adaptation to ICE environments.

\section{Limitations}

This longitudinal field study, conducted in an isolated polar station, has methodological shortcomings inherent in these ecological conditions that limit its generalizability. The serious limitation of the present study is its small sample size, which precludes generalizations and deeper correlational analyses. However, within these intrinsic limitations, our study benefits from standardized, cross-cultural, and multinational approaches recommended in this field (Zimmer et al., 2013). 


\section{Conclusion}

The stressors and consequences observed in the sparse environment of Antarctic winters offer insights into issues that may have impact on space explorations of long duration, such as future missions to Mars. The results of the present study suggest that ICE environments can induce some stress, mainly in social relations, which could lead to subsequent maladaptation. In addition, this study highlights (a) the role of DM in PAPs and its value as a variable to be considered in personnel selection and (b) the importance of Perceived Control in recovery and consequently in adaptation to ICE situations.

A better understanding of the affective, social, and cognitive processes involved in adaptation could help to develop (a) selection to improve adaptation (e.g., including DM assessment in selection process) and (b) psychological countermeasures (e.g., increasing coping repertory) to enhance well-being and to counteract negative psychological sequelae during future longduration space missions. Preventive interventions, including stress management, training in interpersonal relations, leadership skills, and individual CS, should be developed by psychologists and validated in the specific ICE situations for the crew members.

\section{Acknowledgments}

The protocol and parts of this article were presented at the Workshop on Human Behaviour and Performance in Analog Environments and Simulations, ESA/ESTEC, Noordwijk, The Netherlands, August 31, 2010. We would like to thank the winter crew and especially Dr Eoin Mac Donald, the physician in charge to perform the protocol during the wintering.

\section{Declaration of Conflicting Interests}

The author(s) declared no potential conflicts of interest with respect to the research, authorship, and/or publication of this article.

\section{Funding}

The author(s) disclosed receipt of the following financial support for the research, authorship, and/or publication of this article: The study was sponsored by the European Space Agency (ESA) and the French Polar Institute IPEV (Institut Paul Emile Victor). The work reported in this article was funded by research grants from the university and the region of Burgundy and the Centre Nationale d'Etudes Spatiales, France.

\section{References}

American Psychiatric Association. (1994). Diagnostic and statistical manual of mental disorders (4th ed.). Washington, DC: Author. 
Andrews, G. M. D., Singh, M. B. S., \& Bond, M. M. D. (1993). The Defense Style Questionnaire. Journal of Nervous and Mental Disease, 181, 246-256.

Armitage, C. J., \& Conner, M. (2001). Efficacy of the theory of planned behaviour: A meta-analytic review. British Journal of Social Psychology, 40, 471-499.

Bishop, S. L. (2004). Evaluating teams in extreme environments: From issues to answers. Aviation, Space, and Environmental Medicine, 75(7), 14-21.

Bouchard, G., \& Thériault, V. (2003). Defense mechanisms and coping strategies in conjugal relationships: An integration. International Journal of Psychology, 38, 79-90. doi:10.1080/00207590244000214

Brown, K. W., \& Ryan, R. M. (2003). The benefits of being present: mindfulness and its role in psychological well-being. Journal of personality and social psychology, 84(4), 822.

Cheyne, J. A., Carriere, J. S. A., \& Smilek, D. (2006). Absent-mindedness: Lapses of conscious awareness and everyday cognitive failures. Consciousness and Cognition, 15, 578-592. doi:10.1016/j.concog.2005.11.009

Chouker, A. (Ed.). (2012). Stress challenges and immunity in space. Berlin, Germany: Springer. Retrieved from http:/www.springerlink.com/index/10.1007/978-3642-22272-6

Cohen, J. (1988). Statistical power analysis for the behavioral sciences (2nd ed.). Hillsdale, NJ: Lawrence Erlbaum.

Cohen, S. (1978). Environmental load and the allocation of attention. In A. Baum, J. Singer, \& S. Valins (Eds.), Advances in environmental psychology (Vol. 1). Hillsdale, NJ: Lawrence Erlbaum.

Cohen, S., \& Williamson, G. (1988). Perceived stress in a probability sample of the United States. In S. Spacapan \& S. Oskamp (Eds.), The social psychology of health (pp. 31-68). Newbury Park, CA: SAGE.

Cramer, P. (1991). The development of defense mechanisms: Theory, research, and assessment. New York, NY: Springer-Verlag Publishing.

Cramer, P. (1998). Coping and defense mechanisms: What's the difference? Journal of Personality, 66, 919-946.

Erickson, S., Feldman, S. S., \& Steiner, H. (1997). Defense reactions and coping strategies in normal adolescents. Child Psychiatry and Human Development, 28, 45-56.

Fulde, E., Junge, A., \& Ahrens, S. (1995). Coping strategies and defense mechanisms and their relevance for the recovery after discectomy. Journal of Psychosomatic Research, 39, 819-826. doi:10.1016/0022-3999(95)00014-6

Geuna, S., \& Brunelli, F. (1995). Stressors, stress and stress consequences during long-duration manned space missions a descriptive model. Acta Astronautica, 36, 347-356.

Grigor'ev, A. I., \& Fedorov, B. M. (1996). Stresses under conditions of a normal mode of life, during hypokinesia (simulating effects of weightlessness) and in space flights. Fiziologiia Cheloveka, 22(2), 10-19.

Ishizaki, Y., Ishizaki, T., Fukuoka, H., Kim, C. S., Fujita, M., Maegawa, Y., . . G Gunji, A. (2002). Changes in mood status and neurotic levels during a 20-day bed rest. Acta Astronautica, 50, 453-459. 
John Paul, F. U., Mandal, M. K., Ramachandran, K., \& Panwar, M. R. (2010). Cognitive performance during long-term residence in a polar environment. Journal of Environmental Psychology, 30, 129-132. doi:10.1016/j.jenvp.2009.09.007

Kanas, N., Sandal, G., Boyd, J. E., Gushin, V. I., Manzey, D., North, R., . . . Wang, J. (2009). Psychology and culture during long-duration space missions. Acta Astronautica, 64, 659-677. doi:10.1016/j.actaastro.2008.12.005

Kellmann, M., \& Günter, K. D. (2000). Changes in stress and recovery in elite rowers during preparation for the Olympic Games. Medicine \& Science in Sports \& Exercise, 32, 676-683. doi:10.1097/00005768-200003000-00019

Kellmann, M., \& Kallus, K. W. (2001). Recovery-Stress Questionnaire for Athletes: User manual (Vol. 1). Champaign, IL: Human Kinetics.

Lazarus, R. S. (1966). Psychological stress and the coping process. New York, NY: McGraw-Hill.

Lazarus, R. S. (1977). Psychological stress and coping in adaptation and illness. In Z. J. Lipowski, D. R. Lipsi, \& P. C. Whybrow (Eds.), Psychometric medicine: Current trends (pp. 14-26). New York, NY: Oxford University Press.

Lazarus, R. S. (1999). The cognition-emotion debate: A bit of history. In T. Dalgleish \& M. J. Power (Eds.), Handbook of cognition and emotion (pp. 3-19). New York, NY: John Wiley.

Lazarus, R. S., \& Folkman, S. (1984). Coping and adaptation. The handbook of behavioral medicine, 282-325.

Lazarus, R. S. (2006). Stress and emotion: A new synthesis. Berlin, Germany: Springer.

Leon, G. R., Sandal, G. M., \& Larsen, E. (2011). Human performance in polar environments. Journal of Environmental Psychology, 31, 353-360. doi:10.1016/j. jenvp.2011.08.001

Manzey, D., \& Lorenz, B. (1998). Mental performance during short-term and longterm spaceflight. Brain Research Reviews, 28, 215-221.

Marsh, M. S., \& Rygalov, V. Y. (2008). Conceptual approach for stress estimates among astronauts and cosmonauts. Combustion, 2015, 6-22.

Muris, P., \& Merckelbach, H. (1996). The short version of the Defense Style Questionnaire: Factor structure and psychopathological correlates. Personality and Individual Differences, 20, 123-126. doi:10.1016/0191-8869(95)00153-W

Nicolas, M. (2009). Personality, social support and affective states during simulated microgravity in healthy women. Advances in Space Research, 44, 1470-1478. doi:10.1016/j.asr.2009.07.013

Nicolas, M., \& Gushin, V. (in press). Stress and recovery responses during a 105-day ground-based space simulation. Stress and Health. doi:10.1002/smi.2565

Nicolas, M., \& Jebrane, A. (2008a). Consistency of coping strategies and defense mechanisms during training sessions and sport competitions. International Journal of Sport Psychology, 40, 229-248.

Nicolas, M., \& Jebrane, A. (2008b). Relationships between coping strategies and defense mechanisms in sport performance. Psychological Reports, 103, 735-744. doi:10.2466/pr0.103.3.735-744 
Nicolas, M., Sandal, G. M., Weiss, K., \& Yusupova, A. (2013). Mars-105 study: Time-courses and relationships between coping, defense mechanisms, emotions and depression. Journal of Environmental Psychology, 35, 52-58. doi:10.1016/j. jenvp.2013.05.001

Nicolas, M., \& Weiss, K. (2009). Stress and recovery assessment during simulated microgravity: Effects of exercise during a long-term head-down tilt bed rest in women. Journal of Environmental Psychology, 29, 522-528. doi:10.1016/j. jenvp.2009.08.006

Palinkas, L. A. (1992). Going to extremes: The cultural context of stress, illness and coping in Antarctica. Social Science \& Medicine, 35, 651-664. doi:10.1016/0277-9536(92)90004-A

Palinkas, L. A. (2003). The psychology of isolated and confined environments: Understanding human behavior in Antarctica. American Psychologist, 58, 353363. doi:10.1037/0003-066X.58.5.353

Palinkas, L. A., \& Suedfeld, P. (2008). Psychological effects of polar expeditions. The Lancet, 371, 153-163.

Parker, J. D. A., \& Endler, N. S. (1996). Coping and defense: A historical overview. In M. Zeidner \& N. S. Endler (Eds.), Handbook of coping: Theory, research, applications (pp. 3-23). Oxford, UK: John Wiley.

Pearlin, L. I., \& Schooler, C. (1978). The structure of coping. Journal of Health and Social Behavior, 19, 2-21. doi:10.2307/2136319

Salam, A. P. (2012). Exploration class missions on earth: Lessons learnt from life in extreme Antarctic isolation and confinement. In A. Chouker (Ed.), Stress challenges and immunity in space (pp. 425-439). Berlin, Germany: Springer. Retrieved from http://link.springer.com/chapter/10.1007/978-3-642-22272-6_32

Selye, H. (1950). Stress and the general adaptation syndrome. British medical journal, 1(4667), 1383-1392.

Stuster, J., Bachelard, C., \& Suedfeld, P. (2000). The relative importance of behavioral issues during long-duration ICE missions. Aviation, Space, and Environmental Medicine, 71(9, Sect. 2, Suppl.), A17-A25.

Suedfeld, P. (2005). Invulnerability, coping, salutogenesis, integration: Four phases of space psychology. Aviation, Space, and Environmental Medicine, 76(Suppl. 1), B61-B66.

Suedfeld, P. (2010). Historical space psychology: Early terrestrial explorations as Mars analogues. Planetary and Space Science, 58, 639-645. doi:10.1016/j. pss.2009.05.010

Suedfeld, P., \& Weiss, K. (2000). Antarctica natural laboratory and space analogue for psychological research. Environment and Behavior, 32, 7-17. doi:10.1177/00139160021972405

White, R. W. (1959). Motivation reconsidered: The concept of competence. Psychological Review, 66, 297-333. doi:10.1037/h0040934

Zimmer, M., Cabral, J. C. C. R., Borges, F. C., Côco, K. G., \& Hameister, B. R. (2013). Psychological changes arising from an Antarctic stay: Systematic overview. Estudos de Psicologia (Campinas), 30, 415-423. 


\section{Author Biographies}

Michel Nicolas is an associate professor and a psychologist working in the field of environmental, constraining, and extreme psychology at the University of Burgundy, France. His research examines adaptation processes connecting physical environments to human behavior in areas related to both human performance and well-being.

Peter Suedfeld is a professor emeritus of psychology at the University of British Columbia, Vancouver, Canada. His main research interest is human adaptation during and after challenging, stressful, or traumatic environments, using laboratory experiments, field studies, archival content analyses, and questionnaires.

Karine Weiss is a professor in social and environmental psychology in the University of Nîmes, South of France. Her research interests are about adaptation in extreme environments and adjustment to stressful situations. She is also involved in the field of environmental health, with more recent research concerning social representations and behaviors face to chronical and emerging risks.

Marvin Gaudino is pursuing a doctoral degree in the psychology graduate program at the University of Burgundy. His research interests include human environment interactions, mental preparation, and behavior change. 